\title{
La necesaria construcción de una Didáctica específica en Ciencia de la Información. Aportes latinoamericanos a esta discusión
}

\author{
Jimena Núñez Ansuas ${ }^{1}$
}

\section{Resumen}

El objetivo de este trabajo es reunir las propuestas de tres autores cuyos aportes son considerados clave para la construcción de una Didáctica específica en el ámbito de la Bibliotecología y la Ciencia de la Información en Latinoamérica. Por un lado, Josefa Sabor y Ricardo Nassif, docentes argentinos que desarrollaron sus ideas en la década del 60; por otro, Jaime Ríos, docente mexicano que a partir de los 2000 se ha dedicado a investigar la relación de la enseñanza de la disciplina con la definición de su episteme particular. La revisión bibliográfica se centrará principalmente en dos textos: el manual Métodos de enseñanza de la Bibliotecología de 1968 que une los planteos de Sabor con el conocimiento pedagógico de Nassif y Didáctica de la Bibliotecología: Teoría y principios desde la enseñanza de la ciencia publicado por Ríos en 2008. Se considera que para poder entender cómo se enseña en Ciencia de la Información la discusión sobre la conceptualización epistemológica de la disciplina es ineludible. Es decir, arribar a tal conceptualización permitirá diseñar una didáctica específica que acompañe los procesos de su enseñanza. En este sentido, la enseñanza de la bibliotecología ha estado tensionada entre dos perspectivas didácticas, una centrada casi en exclusividad en los aspectos técnicos y procedimentales y una que brega por instalar el estatus epistémico de la disciplina. Eso conlleva a discusiones sobre cómo construir los currículos de grado y posgrado y qué procedimientos de enseñanza se adecuan más a los contenidos y objetivos de los mismos. Se considera que las contribuciones de autores como Sabor, Nassif y Ríos, colaboran en el desarrollo de una didáctica de la Bibliotecología y Ciencia de la Información que equilibre estas tensiones, promoviendo la formación de profesionales que al mismo tiempo que responden a las demandas del mercado laboral, sean críticos y reflexivos.

Palabras clave: Didáctica de la bibliotecología, Educación bibliotecológica - América Latina, Enseñanza de la bibliotecología - América Latina

\footnotetext{
${ }^{1}$ Ayudante en el Departamento Información y Sociedad, Instituto de Información de la Facultad de Información y Comunicación, Universidad de la República (Uruguay). Correo electrónico: jimena.nunez@fic.edu.uy
} 


\title{
The necessary construction of a specific Didactics in Information Science. Latin American contributions to this discussion
}

\begin{abstract}
The objective of this paper is to bring together the proposals of three autors whose whose contributions are considered key to the construction of a specific Didactic in the area of Library Science and Information Science in Latin America. On the one hand, Josefa Sabor and Ricardo Nassif, argentine teachers who developed their ideas in the 1960s; on the other, Jaime Ríos, a mexican teacher who since the 2000s has been dedicated to investigating the relationship between the teaching of the discipline and the definition of his particular episteme. The bibliographical review will focus mainly on two texts: the Manual Methods of teaching of the Library Science (1968) that links the proposals of Sabor with the pedagogical knowledge of Nassif, y Didactics of the Librarianship: Theory and principles from the teaching of science published by Ríos in 2008. It is considered that in order to understand how Information Science is taught, the discussion about the epistemological conceptualization of the discipline is unavoidable. This means that arriving at such conceptualization will allow designing a specific didactic that accompanies the processes of its teaching.In this sense, the teaching of librarianship has been strained between two didactic perspectives, one focused almost exclusively on the technical and procedural aspects and one that struggles to install the epistemic status of the discipline. It carries to discussions on how to build the degree and postgraduate curricula and what teaching procedures are best suited to their contents and objectives.

It is considered that analyzing the contributions of authors such as Sabor, Nassif and Ríos, collaborate in the development of a didactic of Library Science and Information Science that balances these tensions, promoting the training of professionals who at the same time respond to the demands of the labor market, be critical and thoughtful.
\end{abstract}

Keywords: Library science didactics, Library science education- Latin America, Library science teaching - América Latina

\section{Introducción}

El presente artículo reúne los aportes que tres importantes teóricos latinoamericanos han hecho a la discusión sobre la necesidad de construir una didáctica específica para la enseñanza de la Bibliotecología/Ciencia de la Información. Ellos son Josefa Sabor y Ricardo Nassif de Argentina y Jaime Ríos de México. El interés por reunir estas voces, señalando coincidencias y disidencias, se configura como un mojón más dentro de la línea de investigación que quien escribe intenta desarrollar en el ámbito de la enseñanza de la Bibliotecología en la Universidad de la República. 
El punto de partida de este camino fue la inquietud por indagar sobre cómo enseñan los docentes universitarios. Específicamente, se consideró de interés rastrear estudios que existieran sobre las prácticas de enseñanza en Ciencia de la Información en el ámbito latinoamericano. Para ello, nuestro trabajo final de grado consistió en el relevamiento y análisis de las actas de los encuentros de la Asociación de Educación e Investigación en Ciencia de la Información de Iberoamérica en el Caribe (conocida inicialmente como EDIBCIC, actual EDICIC). Ese relevamiento evidenció que muchos docentes e investigadores latinoamericanos aspiran a construir mejores formas de enseñar Ciencia de la Información y para ello se han dedicado a estudiar y analizar las diversas facetas que conforman las tres dimensiones del sistema didáctico. En las conclusiones de este trabajo se señalaron cuatro tensiones de la enseñanza universitaria en Bibliotecología. La primera es la que se da entre la tradicional misión de ser un centro de producción de conocimiento y la demanda social que desde la revolución industrial le exige a la universidad que forme profesionales. La segunda tensión está relacionada con la formación permanente, especialmente en aspectos didácticos y pedagógicos que deberían tener los profesores universitarios. Una tercera tensión puede señalarse entre el diseño de planes y programas y los paradigmas disciplinares, que se hace patente en aquellos análisis de planes de estudio que muchas veces se detienen en descripciones superficiales y no dan lugar a discusiones fundamentales sobre los paradigmas disciplinares. En este sentido, muchos de los trabajos relevados, señalan cómo el énfasis en aspectos técnicos hizo que muchas de las escuelas latinoamericanas no reforzaran las bases epistemológicas de la disciplina, lo que recuerda de cierto modo la tensión entre formación e investigación que se mencionó líneas atrás. La identificación de estos fenómenos hizo que la pregunta inicial sobre cómo se enseña en Bibliotecología/Ciencia de la Información, diera paso a cuestionamientos más profundos que podrían dar lugar a la construcción de una didáctica específica de la disciplina:

Quizás no se deba preguntar únicamente cómo se enseña, sino qué se enseña y para qué se enseña en la universidad, todo ello sin olvidar a quienes enseñan y a quienes aprenden y a la realidad en que sus acciones se desarrollan (Núñez, 2018, p. 87).

Para ello, parece necesario abandonar la idea instalada de que la didáctica es un conjunto de prescripciones normativas que guían la acción educativa. Algunas corrientes de corte instrumental le han adjudicado tradicionalmente un papel prescriptivo. A pesar de que este enfoque ha perdido valor, en la actualidad aún pervive la creencia de que se puede contar con determinadas pautas aplicables en el desarrollo de una situación de clase. Por ello, es que Basabe (Basabe, 2016, p.224) prefiere hablar de principios de procedimientos más que de reglas de actuación y propone, siguiendo a Morin, que la didáctica se piense como una apuesta a la estrategia más que como un programa a seguir.

La didáctica es un cuerpo de conocimiento orientado a guiar la acción educativa, pero la enseñanza, como toda acción social, es siempre 
singular y escapa a su control a partir de reglas. [...] Por ello, exige al destinatario del discurso didáctico, maestros y profesores, reconstruir el significado teórico-práctico de sus enunciados en el marco de la situación particular (Basabe, 2016, p.220).

Así se concibe a la didáctica como una ciencia aplicada donde los conocimientos teóricos (alimentados a su vez por otras disciplinas como la Sociología y la Psicopedagogía) se articulan con la práctica permanentemente. Por ello, la reflexión sobre la acción es útil para conocer mejor la situación de acción didáctica sin obviar las condicionantes que afectan tanto a los sujetos que intervienen como a los saberes culturales en juego. Edith Litwin afirma: "Entendemos a la didáctica como teoría acerca de las prácticas de la enseñanza significadas en los contextos sociohistóricos en que se inscriben" (1998a, p.94). Esto implica que la didáctica se encuentra en permanente transformación y discusión. Es afectada por el estado de los saberes científicos y sociales y por las prácticas y concepciones de enseñantes y aprendientes en determinado momento histórico.

Por lo tanto, otro factor que determina la labor del didacta como profesional de la enseñanza, es que su práctica se desarrolla en una disciplina específica del conocimiento. Históricamente se ha sostenido la existencia de una didáctica general, pero esta se ha encontrado con la dificultad de delimitar su epistemología. Por su lado, quienes defienden la existencia de didácticas específicas lo hacen considerando que cada ámbito de conocimiento y cada nivel de instrucción requiere un estudio particular. La didáctica especial no puede desarrollarse sin considerar el nivel o la disciplina que le da sustento:

Se definen las didácticas especiales como campos específicos de las respectivas ciencias, sin relación con un marco de didáctica general cuya propia existencia se cuestiona, desde la óptica de que la enseñanza siempre opera sobre contenidos de instrucción especializados (Davini, 1998, p.42).

Al estudiar la didáctica en la enseñanza de grado en Ciencia de la Información se deberían tener en cuenta, por lo tanto, dos vertientes de análisis. Por un lado, el nivel universitario en el que se ubica.; por otro lado la disciplina y cómo se enseña. En los apartados que siguen se presentarán conceptos relacionados con la didáctica universitaria y con la didáctica en Ciencias de la Información. Luego, se presentarán los aportes de Josefa Sabor, Ricardo Nassif y Jaime Ríos, docentes que en distintos momentos de la historia de la educación bibliotecológica en Latinoamérica propusieron poner en discusión la construcción de una didáctica específica para nuestra disciplina.

\section{Didáctica Universitaria}

Según la bibliografía consultada (Zabalza, 2002 y 2007; García-Valcárcel, 2001; Behares, 2011) la discusión sobre la necesidad de desarrollar una didáctica universitaria es bastante reciente y está relacionada con el fenómeno de la masificación en el acceso a este nivel de formación. En ese 
debate se cruzan dos posiciones: una que ve como ineludible el desarrollo de esta perspectiva disciplinar, y otra que percibe como un esfuerzo inútil trasladar estrategias provenientes de la educación primaria y media a una institución con objetivos tan distintos como es la universidad.

"Existe la creencia, muy acendrada en la universidad, de que el dominio de los contenidos científicos de la asignatura a impartir constituye la mejor garantía para tener éxito en la enseñanza" (Rosales López, 2001, p. 115). Estas palabras de Rosales López dan cuenta de que la discusión sobre la necesidad de desarrollar una didáctica específica en el ámbito universitario, a pesar de tener larga data, aún no se ha saldado. Behares (2011) señala que desde sus inicios la institución universitaria estuvo asociada a la producción de conocimiento. Sin embargo, desde mediados del siglo XIX, debió incorporar la formación de agentes para cumplir determinadas funciones sociales, es decir la profesionalización. Esa tensión, ya mencionada líneas atrás, dio paso en el siglo XX a una nueva conceptualización de la universidad basada en el ternario universitario. Al respecto, Behares reflexiona:

Del interjuego entre estos tres territorios funcionales de los universitarios surgió la figura del docente universitario: a pesar de que la palabra por su uso más frecuente haga pensar en la enseñanza, esta figura intenta integrar la extensión, la enseñanza y la investigación (Behares, 2011, p.15).

Sin embargo, la preocupación por la enseñanza en sí aparece recién a mediados del siglo XX. Según Behares:

la cuestión de la enseñanza no era problemática para las universidades porque no se la separaba de la producción de conocimiento, y por lo tanto estaba incluida como la 'incorporación del neófito' o iniciante a los programas de producción de conocimiento propios de las universidades" (Behares, 2011, p.18).

Este enfrentamiento entre una visión de la universidad como centro de formación de profesionales o como centro de producción de conocimiento fue salvado, según este autor, de distintas formas. Mientras algunas universidades contaron con dos modelos de docencia, uno destinado exclusivamente a la investigación y otro dedicado a la enseñanza; otras diseñaron los niveles de grado pensando en la formación profesional mientras que el posgrado se dedicaba a la formación de corte más académico, fomentando la investigación y la producción de conocimiento. De todas formas, la didáctica proveniente de la educación básica y media, invadió las aulas universitarias para alimentar la función de enseñanza para la que los docentes parecían no estar formados. Este préstamo de conocimientos entre niveles sin reflexionar sobre la especificidad del ámbito universitario puede llevar a la aplicación de técnicas vacías de contenido:

Sería un error reducir el problema de la formación masificada de profesionales a su dimensión didáctica (vinculada a los sentidos, la programación y la práctica de aula de la enseñanza). El problema se caracteriza mejor incluyendo junto a la preocupación didáctica las 
dimensiones de la selección epistémica de los saberes y sus modalidades, de las funciones que estos saberes tendrán en los trayectos ofrecidos, de la organización curricular, de la administración de la enseñanza, de la formación de enseñantes y de la 'integración de las funciones universitarias (Behares, 2011, p.18).

Si bien en algunas áreas o asignaturas de la universidad, con objetivos más propedéuticos o profesionalistas, la enseñanza puede asumir formas muy similares a las que se supone adopta en la enseñanza básica (se transmiten conocimientos desde un saber instalado); existen espacios de formación que se nutren del encuentro del científico que comparte su práctica investigativa con un estudiante que se asocia (se construyen conocimientos desde un nosaber). Ferreira y Bottentuit (2017) plantean la necesidad de discutir sobre la didáctica en la enseñanza superior, buscando aunar la formación profesional con el fomento de una actitud comprometida con la sociedad y la producción de conocimiento:

[...] el debate acerca de la Didáctica y la práctica de los profesores en la enseñanza superior se inserta como un camino para construir nuevos procesos de enseñanza y aprendizaje capaces de estimular en el alumno el deseo de conocer, el sentido de responsabilidad y el compromiso con el aprendizaje que no se agota en el aula, ya que en cada mensaje reflejado en el aula, en cada texto discutido, en cada seminario planificado y presentado, afloran intencionalidades que pueden llevar a la producción de nuevos valores y prácticas políticas. Al mismo tiempo pueden provocar conflictos instigando el debate, desnudando nuevos escenarios y estimulando nuevas prácticas pedagógicas (Ferreira, M.M., Bottentuit, A.M., 2017, p.22, trad. propia).

En ese sentido, Camilloni visualiza el vínculo de los docentes con el saber didáctico como un espacio de reflexión sobre la episteme del campo disciplinar -qué se enseña-, y sobre la proyección social de su accionar pedagógico -para qué se enseña en la universidad-, que no puede reducirse jamás a "presentarles el saber didáctico disciplinario a través de una versión enseñable y adaptada a una rápida transmisión" -cómo se enseña-. En ese espacio de reflexión los docentes desarrollan capacidades de traducción de los principios didácticos en un "proyecto y una práctica pedagógica" (Camilloni, 2007, p.41). Esa práctica pedagógica leída como un encuentro entre quien enseña y quien aprende, permite la construcción de ciertos contenidos culturales, seleccionados del universo más amplio de contenidos por enseñar. La idea de "configuraciones didácticas" (Litwin, 1998b) colabora con el análisis de las prácticas. Para esta autora, cada docente tiene una forma particular que va desarrollando en el transcurso de ese encuentro con el estudiante, con la intención de favorecer el aprendizaje de aquel conocimiento que se está compartiendo.

[La configuración didáctica] implica una construcción elaborada en la que se pueden reconocer los modos en que los docentes abordan múltiples temas de su campo disciplinario y que se expresan en el tratamiento de los contenidos, el particular recorte de los mismos, los supuestos que maneja respecto del aprendizaje, la utilización de 
prácticas metacognitivas, los vínculos que establece en la clase con las prácticas profesionales involucradas en el campo de la disciplina de que se trata, el estilo de negociación de significados que genera, las relaciones entre práctica y la teoría que involucran lo metódico y la particular relación entre el saber y el ignorar (Litwin, 1998b, p.51).

Existen, por su parte, configuraciones no didácticas "que implican sólo la exposición de ideas o temas, sin tomar en consideración los procesos del aprender" (Litwin, 1998b, p.51).

Por ello, se considera ineludible generar procesos reflexivos sobre las prácticas de enseñanza en el escenario de la educación superior. Sin embargo, esto no significa invadir las clases con conceptualizaciones didácticas. Behares plantea el riesgo de caer en el didactismo desconociendo el bagaje y experiencia que los docentes universitarios portan. Así, la reflexión que hace el docente sobre su propia formación pedagógica y sobre sus prácticas cotidianas permitiría la generación de conocimiento sobre la didáctica de su disciplina.

\section{Didáctica de la Bibliotecología / Ciencia de la Información}

La Ciencia de la Información busca afirmarse a lo largo del siglo XX a partir de la ampliación de su campo y objeto de actuación, antes restringido a las bibliotecas y a los libros. La construcción gradual de su teoría ha debido luchar contra el pragmatismo y eso se ha visto reflejado en la formación profesional. Así, la formación en aspectos técnicos y procedimentales ha dejado de lado muchas veces la construcción y el debate de sus principios epistemológicos y su posterior realización en la práctica profesional.

Discutir la Bibliotecología en el sentido de aplicar a los niveles de conocimiento comprensión, análisis, síntesis y evaluación entre teoría y práctica, requiere la construcción de un referencial científico, que ayude a desarrollar criticidad, es decir, no absorber pasivamente los conocimientos, sino reaccionar con estímulos internos, buscando constituirse como ciencia social cuyos intereses están imbricados con la necesidad de transformar la realidad social [...] (Ferreira, M.M., Bottentuit, A.M., 2017, p.24, trad. propia).

En varios de los documentos analizados para nuestro trabajo final de grado se hace referencia a que en el ámbito de la enseñanza de la Bibliotecología existen ciertas prácticas arraigadas que cierran el paso a la generación de posturas críticas en los estudiantes que permitirían crear nuevos conocimientos. La apertura a nuevas metodologías de enseñanza y la discusión sobre las concepciones que los docentes portan sobre lo que es el aprendizaje podría "estimular la enseñanza de la Bibliotecología dentro de contextos en los que los alumnos puedan sentir voluntad y deseo de interferir" (Ferreira, M.M., Bottentuit, A.M., 2017, p.24, trad. propia).

Una posible explicación a este fenómeno puede deberse a que la formación que portan los docentes ha sido excesivamente tecnicista y carece o tiene escasos contenidos de corte pedagógico. En este sentido, el desarrollo de 
una didáctica disciplinar puede brindar herramientas para que los docentes lleven adelante prácticas de enseñanzas transformadoras. Según Stoker (2000) la enseñanza de la Ciencia de la información se encuentra en proceso de cambio continuo.

Quizás el cambio más importante ha sido el reconocimiento universal de que la educación profesional debe ser un proceso continuo. Los conocimientos y habilidades adquiridas tendrán una vida relativa y deberán ser revisados y renovados con frecuencia. Lo máximo que se puede esperar de la educación en Bibliotecología y Ciencia de la Información es la provisión de un punto de referencia y fundamento sobre el cual construir una base de conocimiento individual creciente y un rango de competencias (Stoker, 2000:p.121, trad. propia).

\section{Enseñanza de la Bibliotecología en Latinoamérica}

La enseñanza de la profesión bibliotecológica se inició en América Latina y el Caribe a comienzos del siglo XX. En 1909 fue ofrecido el primer curso en Argentina en el ámbito de la enseñanza superior no universitaria. Es en 1922 que se crea una escuela en el ámbito de la Facultad de Filosofía y Letras de la Universidad de Buenos Aires.

En México, los primeros cursos de capacitación para el personal de bibliotecas y archivos aparecen en 1912, también fuera de la esfera universitaria. Luego de depender de secretarías de instrucción y educación pública en forma de escuelas (1916, 1925-1928, 1945), se establece el Colegio de Bibliotecología de la Universidad Nacional Autónoma de México (1956).

En la década del 70 se contaba con más de 60 escuelas distribuidas en los países latinoamericanos, 28 en Brasil, 13 en Argentina, Colombia y México con 3, Costa Rica y Venezuela tenían 2 y el resto de naciones solamente una. Judith Licea (1975:154) realiza en ese momento un diagnóstico de la realidad bibliotecológica de la región, señalando la falta de personal idóneo en los servicios de información. Según esta autora mexicana eso podía explicarse analizando el funcionamiento de las diversas escuelas. Al hacerlo halló que los planes y programas de estudios eran copias de instituciones extranjeras, en particular norteamericanas y europeas, y que los métodos y técnicas de enseñanza separaban al estudiante de la realidad.

En suma, percibe que en el diseño de los planes y programas de estudios se debe tomar en cuenta el contexto de la realidad nacional para avanzar en el planteamiento de soluciones referentes a los problemas que padecen estos países. Sugiere así la necesidad de que en materia de métodos y técnicas de enseñanza se estime la unidad teoría-práctica, sea cual fuere el punto de apoyo respecto del conjunto de actividades que realizan los profesionales de la Bibliotecología (Meneses Tello, F., 2010, p.15).

Asimismo, Licea plantea que las escuelas de Bibliotecología latinoamericanas deben estar atentas a las constantes transformaciones que afectan a los servicios bibliotecarios locales. El docente que forme a los 
futuros profesionales deberá tener una mirada integral y comunitaria:

La meta de este profesional debe ser, por lo tanto, servir a la sociedad mediante nuevas perspectivas de enseñanza y aprendizaje, con el fin superior de formar cuadros profesionales de la bibliotecología, idóneos para enfrentar los retos que nos desafían en esta región (Meneses Tello, F., 2010:16).

\section{Aportes latinoamericanos a la construcción de una Didáctica específica de la Disciplina}

Si bien los autores estudiados en el presente trabajo tienen en común haber desarrollado su actividad docente en escuelas de Latinoamérica, es claro que los cincuenta años que separan a Sabor y Nassif del mexicano Ríos influyen en sus apreciaciones. Mientras los primeros observaban con cautela el advenimiento de las tecnologías de la información y advertían sobre los riesgos de que la Bibliotecología diera paso a la Documentación; Ríos se instala en un escenario donde las TIC han ganado terreno, y donde la Ciencia de la Información ha ido fortaleciéndose en su construcción epistemológica. Sin embargo, en los textos que se analizan pueden señalarse líneas temáticas en común que permiten ensayar una comparación que dé cuenta de las coincidencias y disidencias entre ellos. Para definir esas líneas temáticas se decide retomar las tensiones identificadas en nuestro trabajo final de grado ya mencionadas en la introducción, páginas atrás.

\subsection{Josefa Sabor y Ricardo Nassif}

Josefa Sabor nació en España en 1916 obteniendo en 1937 la nacionalidad argentina. Se especializó en la Facultad de Filosofía y Letras de la UBA en Historia y Biblioteconomía. En 1938 ingresó como asistente a la biblioteca del Instituto de Enseñanza de la UBA. Entre 1943 y 1946 fue Directora de la Biblioteca del Instituto de Bibliografía y Biblioteconomía de la UBA. Luego dirigió la biblioteca del Museo de Ciencias Naturales. Desde 1955 hasta 1964 fue directora de la Biblioteca Central de la Facultad de Filosofía y Letras de la UBA. Fue docente en diversas universidades, asesora de organismos internacionales e investigadora del CONICET hasta 1980. Fue participante y pionera en el proceso de profesionalización de la Bibliotecología en Argentina. En 1968 publicó el manual Métodos de enseñanza de la Bibliotecología en el que unió su experticia disciplinar con el conocimiento pedagógico de Ricardo Nassif, profesor de la Universidad Nacional de La Plata. En el prefacio de la edición que realiza Unesco se plantea que la cantidad de escuelas existentes no responden adecuadamente a la demanda de personal y se señala, a su vez, que hay falta de profesores. En ese momento, quienes enseñan son los que han trabajado en diversas bibliotecas: "Este personal, pese a su experiencia y capacidad, no tiene, en la mayor parte de los casos, una preparación pedagógica adecuada y esto limita, en cierta medida, la calidad de la enseñanza que imparte" (Sabor, 1968). Ese texto aspiró en su momento a colaborar en la mejora de la enseñanza de la bibliotecología, ofreciendo orientaciones y métodos para el dictado de las clases. 
La propuesta de Sabor sobre los métodos de enseñanza de la bibliotecología se nutre de los aportes que Nassif realiza en su estudio preliminar. Ricardo Nassif fue maestro y egresó como Licenciado en Filosofía y Ciencias de la Educación de la Universidad de La Plata. Su producción más prolífica se corresponde a su ejercicio como profesor de Pedagogía en esa misma facultad, entre 1956 y 1975. Escribió, en 1958, el libro Pedagogía general, al que le siguieron otros sobre los aportes al campo de pensadores como Dewey, Martí, Spranger, Rousseau, Krieck, entre otros. Se ocupó de una gran variedad de temas: pedagogía universitaria, educación americana y argentina, educación contemporánea, educación media, currículo, enfoque económico de la educación. Es por ello, que se considera pertinente analizar también las reflexiones que Nassif aporta al trabajo de Sabor.

\subsection{Jaime Ríos}

Jaime Ríos nació en México en 1960 y es investigador del Instituto de Investigaciones Bibliotecológicas y de la Información (IIBI) de la UNAM, del cual fue su primer Director. Es doctor en Bibliotecología y Estudios de la Información por la UNAM. Sus líneas de investigación son: didáctica de la Bibliotecología, evaluación de revistas académicas, normalización y control bibliográfico y el rol social del bibliotecólogo. En 2008 publica Didáctica de la Bibliotecología: Teoría y principios desde la enseñanza de la ciencia y en 2015 compila junto a Pulido Daza, Didáctica de la bibliotecología: aproximaciones e instituciones.

En la primera publicación referida, Ríos plantea que su investigación

nació de la preocupación por construir y organizar los fundamentos de la didáctica bibliotecológica, la cual reconoce como punto de partida la complejidad de la bibliotecología, así como los problemas fundamentales, tanto teóricos como prácticos, que giran en torno a su enseñanza (Ríos, 2008, p.vii).

\subsection{Primera tensión: Universidad como centro de producción de conocimiento / énfasis en la formación de profesionales para el mercado laboral}

Sabor señala que la discusión sobre el peso que deben tener en la formación los aspectos teóricos y prácticos no es nueva, sino que se remite a los primeros años del siglo XX. Según la época y los países, las escuelas han fluctuado entre una y otra tendencia. Quienes apoyan el mayor peso de la práctica aducen que los egresados no pueden salir sin una base realista pues eso los obliga a formarse en el propio ejercicio profesional. Mientras tanto, los que defienden una mayor presencia conceptual consideran que esta es la que dota de mejores armas al profesional para que enfrente situaciones novedosas e impredecibles.

Es necesario que el bibliotecario sea capaz, en una situación dada, de reaccionar eligiendo el mejor camino y llegando a la solución más justa, no tanto porque se le haya enseñado ese caso en particular, cuanto porque conoce teorías y principios y tiene la suficiente 
seguridad de juicio para adaptarlos y aplicarlos a cualquier tipo de situación (Sabor, 1968, p.82).

Según la autora, si lo que se quiere es fomentar una actitud crítica y creativa para el futuro desempeño profesional, la enseñanza de la bibliotecología debe no solo hacer énfasis en los fundamentos teóricos de la disciplina, sino que también debe ofrecer contenidos de orden general:

En ese sentido parece indispensable una base humanista, que dé a la mentalidad del bibliotecario amplitud, flexibilidad, posibilidades de síntesis y generalización y, sobre todo, sirva de apoyo a una ética que también debe formar parte del bagaje profesional (Sabor, 1968, p.83).

En ese momento, Sabor distinguía dos señales de que este tipo de formación era urgente: la pobreza de la investigación bibliotecológica y el escaso número de profesionales en los cargos de jerarquía de las unidades de información.

Por su lado, Nassif plantea la necesidad de que la Universidad problematice sus contenidos. Este autor identifica doce temas o problemas propios de la pedagogía bibliotecológica. Aquí nos centraremos en el que se refiere a la "metodología de la enseñanza bibliotecológica, según los objetivos de la doble formación teórica y técnica" (Nassif, 1968, p.30). Este pedagogo considera necesario definir el método didáctico como una especie particular dentro del método educativo cuyo objeto es la "dirección del aprendizaje", dejando de lado la visión clásica de ser un "cuerpo rígido de métodos" que imponen al alumno "un saber elaborado de antemano" en cuya elaboración ni docente ni estudiante ha participado (Nassif, 1968:37). En el momento en que Nassif teorizó sobre estos temas, se estaba dejando de lado la concepción del aprendizaje como un acto de retención y repetición y se lo entendía como "toda actividad mental de que es capaz el alumno en relación con el manejo y la elaboración de los materiales o contenidos formativos" (Nassif, 1968, p.39). En este sentido, el autor enumera múltiples actividades que no se basan en la memoria o en la simple asimilación de contenidos sino que apelan a la construcción, discusión y ejecución de experiencias.

Al centrarse en el estudio del método en la enseñanza superior, Nassif es consciente de los peligros que acarrea instalar una pedagogía sin tener en cuenta lo que enseñantes y aprendientes ya están elaborando en conjunto en las aulas universitarias:

[...] las dificultades tendrán fuerza si hacemos de la pedagogía universitaria y de su respectiva didáctica un recetario de normas rígidas e inviolables capaces de aniquilar la energía creadora de los individuos que enseñan y aprenden en la universidad, y hasta la misma riqueza de los bienes culturales con los cuales ella trabaja (Nassif, 1968, p.40).

Este fenómeno fue analizado en nuestro trabajo final de grado, al encontrar que varios docentes manifestaban en sus ponencias una gran preocupación por el escaso registro que de sus decisiones didácticas hacían. Eso provoca 
una pérdida de experiencias que podrían enriquecer las prácticas de enseñanza de otros profesores. La sistematización de las prácticas no solo permite su evaluación y replicación sino que también sirve de base para la generación de teorías. No registrar y sistematizar esas experiencias constituye, en palabras de Santos (2010), un "epistemicidio". Esa energía creadora de docentes y estudiantes que menciona Nassif, es la que define la esencia de la universidad como "centro de problematización y búsqueda" (Nassif, 1968, p.41) y la diferencia de niveles de formación inferiores:

[...] la universidad se define por tender a una problematización permanente de sus contenidos, posibilitada por la madurez de los estudiantes, con el agregado de que aquella se produce por intermedio de la búsqueda en común. La universidad se sostiene por la labor conjunta de profesores y alumnos. Sin esa comunidad de trabajo no hay universidad [...] (Nassif, 1968, p.42).

Esta referencia a la problematización de contenidos da pie al análisis de la díada enseñanza/investigación que forma parte del permanente debate entre una visión de la universidad como centro de formación de profesionales o como centro de producción de conocimiento. Nassif coincide con Behares al identificar espacios de formación que se nutren del encuentro del científico que comparte su práctica investigativa con un estudiante que se asocia. $\mathrm{Si}$ definimos a la enseñanza superior como problematizadora de sus contenidos, esto supone "una reelaboración y un análisis crítico, análisis del cual debe ser capaz no sólo el profesor, sino el alumno estimulado y dirigido por aquél”' (Nassif, 1968, p.43).

Ríos, por su parte, considera que la formación de los estudiantes debe nutrirse fuertemente de las bases teóricas de la disciplina pero no para que sean únicamente investigadores que creen nuevos conocimientos, sino para que cuenten con herramientas que les permitan analizar y solucionar los fenómenos y problemas sociales que la realidad les pondrá enfrente. Es en este sentido que Ríos defiende la necesidad de visualizar a la Bibliotecología como una disciplina científica. Dentro de ella, identifica dos niveles de teorización. La teoría explicativa se ha enfocado a la construcción de un corpus conceptual que permita explicar los fenómenos que son su objeto de estudio. La teoría prescriptiva, conceptualiza a partir de situaciones prácticas, proporcionando normas y recomendaciones tendientes a la acción social. Sin embargo, dentro de las teorías prácticas o prescriptivas, identifica dos niveles de complejidad. Las básicas son aquellas de poco alcance, relacionadas con ámbitos de acción como la adquisición, la catalogación, la conservación documental, la indización, entre otras. Las generales, en cambio, proponen prescripciones comprensivas, que apuntan a su utilidad social: “[...] estas teorías recomiendan lo que debería hacerse y suponen un compromiso deliberado por parte del teórico; esto es, con alguna finalidad que debe buscarse y adoptarse" (Ríos, 2008, p.5).

El autor considera necesario especificar que detrás de toda práctica existe una teoría: 
La práctica bibliotecológica que concreta las teorías prescriptivas generadas en la disciplina, no se reduce a un conjunto de rutinas o cadenas operativas de trabajo; las prácticas bibliotecológicas operan sobre la base de una teoría que incluye fines deseables como metas y los medios para lograrlas; si no contara con estos elementos sólo se estarían manifestando conductas sin sentido y la actuación no sería en realidad la ejecución de una práctica (Ríos, 2008, p.8).

Ríos releva críticamente distintas visiones que niegan a la Bibliotecología su capacidad de constituirse como disciplina científica para luego reafirmar su postura en contrario:

Ante este panorama considero necesario destacar una perspectiva que reconozca la naturaleza teórica de la bibliotecología, independientemente de que se piense en ella como centrada en el "oficio" o en la "práctica"; o bien, referimos a ella como ciencia. Ya sea que se trabaje en función de ideales explicativos o de que se atiendan necesidades sociales, en ambos casos se resuelven problemas de carácter cognoscitivo o se responde a requerimientos sociales. Por tanto, en la bibliotecología se abordan dos tipos de problemas cuya solución requiere de una teoría explicativa o prescriptiva. Pero en ningún caso la teoría puede estar disociada (Ríos, 2008, p.15).

Otra revisión que realiza Ríos es la de los planes de estudio de Bibliotecología vigentes en México a principios de los 2000. Concluye que, en ese momento, predominaba el enfoque técnico que privilegia la formación en el desempeño profesional. Sin embargo, reconoce esfuerzos como los de Morales y Lafuente en 1992, en cuya propuesta curricular se ve que:

la organización teórica de la disciplina sirve como criterio para diseñar un currículum y ponen el énfasis en la enseñanza de los principios y teorías, así como en otras disciplinas a la vez que le conceden un interés fundamental a la enseñanza de estas entidades (Ríos, 2008, p. 24).

Esta forma de plantear la enseñanza de la disciplina pretendía que el alumno se pudiera apropiar de conocimientos que le permitan, además de identificar fenómenos, vincularlos con sus aplicaciones prácticas. A pesar de esta propuesta, los siguientes planes curriculares se supeditaron a la realidad profesional que se vivía dentro de las bibliotecas y recurrieron escasamente a la disciplina bibliotecológica. El autor advierte que si los planes de estudio continúan fortaleciendo los aspectos relacionados a la actividad profesional, la Bibliotecología perderá estatus académico frente a otras disciplinas con las que convive dentro de sus facultades.

Es importante, pues, que se fortalezca equilibradamente la enseñanza de la disciplina, tanto por lo que toca a sus ideales explicativos -como disciplina teórica-, como por lo que se refiere a la enseñanza del conocimiento práctico de la disciplina (Ríos, 2008, p.42).

Ese equilibrio también reclamado por Sabor, se podría alcanzar construyendo una didáctica disciplinar: 
que permita superar la concepción profesionalizante o academicista de la enseñanza e incorpore los avances que se han dado tanto en el campo educativo, como en la enseñanza de la ciencia y en las Humanidades (Ríos, 2008, p.42).

En su publicación del 2015, Ríos retoma la relación teoría y práctica y su tratamiento en la didáctica disciplinar que propuso en 2008. Señala que no es su intención que el aula se transforme en una imitación de contextos de investigación, alejándose así de los problemas empíricos y profesionales:

La bibliotecología como profesión está estrechamente vinculada a la solución de problemas sociales y quitar este conocimiento profesional a nuestros estudiantes, es claramente suicida. [...] En lo fundamental se propone obrar de acuerdo con el argumento siguiente: toda vez que el estudiante tiene dominio de la racionalidad que subyace en el conocimiento disciplinario, tanto teórico como práctico, cuenta con mayores garantías de interactuar eficaz y exitosamente con la realidad, ya que posee la capacidad racional para confrontar los problemas prácticos que se presentan en el campo profesional, o bien, para transformar o modelar nuevos contextos (Ríos, 2015, p.7-8).

Este dominio de la racionalidad científica subyacente al conocimiento disciplinar, otorga al estudiante autonomía al momento de generar nuevo conocimiento. tanto en el aula como en su futuro desempeño laboral. En ese sentido, Nassif realiza una distinción entre el tratamiento de los saberes culturales que se hace en los niveles primarios y secundarios de la enseñanza institucionalizada y el que se propone realizar la universidad. Si bien sus afirmaciones pueden contradecir las actuales teorías didácticas en primaria y secundaria, se puede acordar que en los niveles iniciales el alumno básicamente recibe destrezas y elementos culturales que le permiten desenvolverse en sociedad y se le muestran (se le enseñan) los resultados de las ciencias y las artes. Mientras tanto, en la educación superior:

la enseñanza reproduce el proceso de formación de los conocimientos, de las doctrinas o de las obras. [...] la enseñanza se convierte en el medio de que se vale el profesor para que el alumno llegue a apropiarse del método de trabajo creador (Nassif, 1968, p.44).

Ya en 1968, Nassif plantea la necesidad de asumir una postura frente "al difícil problema que plantea el inusitado crecimiento y diversificación de la cultura." Al ser imposible transmitir todos los conocimientos, la enseñanza universitaria debe "preparar al sujeto para que los adquiera por sí mismo, desarrollando sus capacidades básicas para elaborarlos y alcanzarlos" (Nassif, 1968, p.44-45).

\subsection{Segunda tensión: Formación en aspectos didácticos y pedagógicos que deberían tener los profesores universitarios}

Con respecto a la necesidad de pensar una pedagogía a nivel universitario, vale rescatar las reflexiones que realiza Ricardo Nassif en el primer apartado del manual. Páginas atrás, se citaba a Rosales López (2001), quien considera 
que la instalada creencia de que la mejor garantía de una enseñanza exitosa es el dominio de los contenidos científicos, no da lugar al desarrollo de una pedagogía y una didáctica universitaria. Las palabras de Nassif (1968) coinciden plenamente con esa reflexión:

La segunda dificultad que se opone al reconocimiento de un lugar primordial a la pedagogía en el análisis y solución de las cuestiones de la enseñanza superior, suele encontrarse en la propia universidad y reviste la forma de un 'prejuicio antipedagógico' muy común en los círculos académicos, tanto humanísticos como científicos. Los docentes universitarios -que sin duda lo son en virtud del completo dominio de un sector cultural- creen que el solo saber especializado es más que suficiente para cumplir la tarea docente en el ciclo superior, y que la individualidad erudita y experimentada está por encima de los métodos didácticos y los sistemas pedagógicos (Nassif, 1968, p.28).

Ante esta resistencia, Nassif recuerda que la universidad es esencialmente una comunidad educativa y que por ello "reclama un severo examen pedagógico" (Nassif, 1968, p.29). Según el autor, la pedagogía universitaria debe dejar su carácter empírico e intuitivo (recordando la didáctica del sentido común que describe Camilloni, 2007) para pasar a ser científica y sistemática. Los docentes deben comprometerse con un estudio y análisis de la realidad universitaria en la que accionan: desde la organización, el rol de la investigación, los planes de estudio, las formas de aprendizaje de los alumnos, hasta la realidad social y cultural de la comunidad en la que se inserta.

Con relación a los procedimientos de enseñanza, tanto Sabor como Nassif coinciden en un enfoque fuertemente instrumental. A pesar de que Sabor acuerda con una forma de enseñanza que propicie la actitud crítica y creativa de los estudiantes, el listado que realiza de los métodos de enseñanza se asemeja mucho a las cartas descriptivas que critica Díaz Barriga en los años 80. Esas cartas se proponían "identificar la conducta observable deseada y establecer las condiciones en que se muestra la conducta y los criterios de realización aceptable" (Díaz Barriga, 1984, p.21). Entre sus propuestas pueden señalarse: el procedimiento expositivo (lecciones), las discusiones en clase, entrevistas y visitas de bibliotecarios expertos, asistencia a conferencias, visitas, trabajos prácticos o laboratorios, prácticas en bibliotecas, estudio de problemas y casos, elaboración de monografías y bibliografías y, por último, realización de seminarios. Este listado, creciente en complejidad e implicancia de los estudiantes en su construcción, también es compartido por Nassif. Él parte del procedimiento expositivo de corte magistral, pasa por la conversación y discusión y llega a las formas de experiencia directa, las llamadas prácticas de "laboratorio". La forma más elevada para este autor es el seminario: "donde se hace el aprendizaje de la investigación científica mediante la aplicación del método específico que cada ciencia emplea para establecer sus verdades y conclusiones" (Nassif, 1968, p.58).

Ríos también hace énfasis en que el desarrollo de los fundamentos de una didáctica específica depende de las categorías de racionalidad de la ciencia 
bibliotecológica. Sin embargo, le preocupa mucho más atender a los procesos de aprendizaje poniendo el foco en quienes aprenden y cómo lo hacen y no solo únicamente en cómo se enseña. Para ello, realiza un breve recorrido histórico sobre el concepto de didáctica. Analiza algunas propuestas que influyeron en su momento en los modelos de enseñanza, como la tecnología educativa de los 70 que basándose en el conductismo presentó, según él, una riesgosa simplificación de las prácticas educativas. Esa propuesta que se centraba en un diseño instruccional que proponía utilizar técnicas e instrumentos, terminó, según el autor, "por suprimir la realidad sobre la cual tenían intención de incidir" (Ríos, 2008, p.55). Al mismo tiempo, considera esencial tener presentes las distintas teorías sobre el aprendizaje. Rescata la de Tyler, donde el centro de las iniciativas de enseñanza se corre del maestro hacia el alumno. Este modelo pretende formar al estudiante desde su interior, a través de la experiencia y teniendo en cuenta sus conocimientos previos. Ríos señala que al prestarse atención a esta variable, las didácticas especiales pasan a ser sustituidas por la didáctica de la ciencia:

De igual modo, el saber previo de los alumnos se transformará en un problema de investigación denominado concepciones previas, y la significación se trabajará en función de la historia de la ciencia y el cambio conceptual, y en el contexto del cognoscitivismo (Ríos, 2008, p.64).

Más allá de la disciplina que se esté enseñando, la didáctica de la ciencia "abordará los problemas relativos a la representación del conocimiento en el individuo y en las comunidades epistémicas, y el cambio conceptual en ambos" (Ríos, 2008, p.64). La didáctica deberá propender a transformar las concepciones previas que traen los alumnos en conocimiento científico más complejo, para que pueda generar nuevas formas de leer la realidad.

\begin{abstract}
Durante la escolaridad, los alumnos han de formarse una determinada concepción sobre lo que es la ciencia, no sólo como cuerpo de conocimientos, sino como manera de pensar el mundo y de construir explicaciones. Se tendría entonces, que la función de la enseñanza, en la didáctica de las ciencias, es la de facilitar la evolución y transformación de las concepciones de los alumnos hacia concepciones científicas más elaboradas (Alvarado y Flores 2001, citado en Ríos, 2008, p.71).
\end{abstract}

Ríos señala varias tensiones que dificultan la puesta en práctica de una didáctica de la Bibliotecología. La primera sería "la desvinculación del conocimiento bibliotecológico que se enseña con respecto a su origen disciplinario y su construcción, validación y organización racional" (Ríos, 2008, p.79). La segunda, la falta de formación de los docentes, tanto en los aspectos disciplinares como en los pedagógicos, especialmente en lo que se refiere a "cómo el aprendizaje del conocimiento teórico y práctico de la disciplina es construido por los alumnos e incluso los propios profesores" (Ríos, 2008:79).

A partir de estas reflexiones, el autor plantea que: 
la enseñanza de la bibliotecología deberá ser capaz de: a) Promover el aprendizaje de las representaciones acerca de los supuestos centrales y los principios de organización de una materia. b) Generar explicaciones fructíferas sobre los fenómenos de la disciplina. Además, deberá formar a sus estudiantes para desarrollar preguntas que tengan relevancia cognoscitiva (Ríos,2008, p.80).

Para el aprendizaje propone tres dimensiones: al abordaje de teorías bibliotecológicas, la justificación de ese conocimiento y la posterior crítica del mismo. Se promueve de esa manera el pensamiento crítico en el alumnado: capacidad de análisis, evaluación y contrastación de argumentos. Desarrollar estas competencias en el estudiante y futuro profesional alimentarán la creación de nuevas teorías disciplinares.

En su publicación del 2015, Ríos vuelve a ocuparse de este tema, teniendo en cuenta las recomendaciones de la UNESCO sobre la educación superior:

[...] Entre estas recomendaciones destaca la necesidad de formar estudiantes con un fuerte compromiso humanista, social y político. Asimismo, pone de relieve el hecho de que sus capacidades deben ser eminentemente reflexivas, críticas y con sólidos conocimientos tanto técnicos como tecnológicos. Esto es un reto complicado, pues el énfasis puesto en el razonamiento de ningún modo excluye el imperativo de contar con el conocimiento técnico y tecnológico de la disciplina (Ríos, 2015, p.5).

En ocasión del XI EDICIC organizado por la Escuela Interamericana de Bibliotecología de la Universidad de Antioquia, el profesor Jaime Ríos realizó una presentación titulada Vindicación de la didáctica de la bibliotecología: construir la racionalidad y el sentido en una disciplina compleja. Esta conferencia asumió una estructura narrativa donde Ríos fue desplegando las distintas etapas que llevaron a la humanidad a generar las disciplinas de la Archivología y Bibliotecología con el fin de conservar su memoria colectiva. El desarrollo disciplinar provocó que quienes la llevaban adelante vieran necesario concebir una forma de transmitir el oficio, lo que dio paso a la construcción de didácticas específicas. Ríos entiende que ese modelo de formación básico para los aprendices debe tener como objetivo que ellos aprendan:

el lenguaje de cada disciplina, las preguntas relevantes y los problemas significativos que le son propios, las entidades teóricas de que se compone su disciplina, los esquemas explicativos básicos y sus textos canónicos, los criterios de credibilidad que juzgan las aportaciones, los paradigmas de experimentación y de observación así como sus criterios de confiabilidad y los conjuntos de razonamientos lógicos y justificados de la Bibliotecología y la Archivología (Ríos, 2018).

La sugerencia de este autor en cuanto a la metodología didáctica más adecuada para lograr estos aprendizajes se resume en dos conceptos: el pensamiento abductivo y la razón ampliada. La forma de aplicarlos en el 
encuentro con los estudiantes en el aula requiere, en primer lugar, de la generación de un nicho cognitivo, trabajo que realizan en conjunto docente y alumnos. Este nicho está basado en las teoría y los conceptos y los modos de explicar los fenómenos con los que los estudiantes van abordando su disciplina. Esta labor provoca dos efectos. Por un lado, ese conjunto de explicaciones le enseña al alumno a ver el mundo desde esa disciplina, les enseña a comprender esos fenómenos con los que va a interactuar. Por otro lado, se busca que el estudiante, a partir de las teorías y conceptos que domina, logre dar sentido y soluciones a ese mundo con el cual va a interactuar. De lo que se trata, finalmente es de que aprenda a traducir los hechos y los fenómenos que observa en preguntas para poderlos resolver. Esas preguntas tienen que ser "cognoscitivamente relevantes" y de igual modo las respuestas "se suponen que son ampliativas en el sentido de que proponen nuevas luces para entender el fenómeno" (Ríos, 2018).

Con relación a la necesidad de que los docentes de Bibliotecología cuenten con formación pedagógica, Ríos aspira a que ellos posean no solamente un sólido conocimiento teórico y práctico de la misma, sino también una fuerte formación en los formatos de aprendizaje y construcción del conocimiento de los estudiantes. Del mismo modo, se espera de ellos el compromiso en querer generar el aprendizaje. El autor señala que los métodos y tecnologías de la enseñanza son auxiliares y que lo realmente sustancial son las concepciones de ciencia, conocimiento y aprendizaje sobre las cuales se instrumentan.

Ineludiblemente, el docente debe transformar sus propias concepciones epistemológicas, disciplinarias y sobre el aprendizaje" (Ríos, 2015, p.11). En ese sentido, Ríos, espera que el docente se comprometa en relación al conocimiento disciplinar y formativo. Sin embargo, contar con una sólida teoría en la cual apoyarse no garantiza el éxito en la enseñanza ni en los aprendizajes. El docente debe estar dispuesto a revisar sus prácticas: "sería una fantasía muy cómoda creer que se puede mejorar la práctica formativa sin tener que transformar el pensamiento y dejar en paz lo aprendido (Ríos, 2015, p.12).

Sin embargo, Ríos identifica que el mejoramiento de la labor docente se ve obstaculizado por varios elementos. El primero a destacar es:

la falta de formación y actualización docente en buena parte de la planta académica de las escuelas, lo cual se explica por su incorporación temprana a la academia sin la exigencia de certificación profesional para la enseñanza de la disciplina (Ríos, 2015, p.13).

A este se suman otros: la real vocación o motivos de elección de la carrera por parte de los estudiantes, la permanente discusión entre la calidad científica o meramente profesional de la disciplina y los estereotipos laborales. Estos factores "integran en su conjunto un contexto conflictivo de aprendizaje, el cual no favorece el aprovechamiento académico, como tampoco permite enriquecer el trabajo docente" (Ríos, 2015, p.13). 


\subsection{Tercera tensión: Importancia de los paradigmas disciplinares en el diseño de planes y programas}

De los temas de la pedagogía bibliotecológica que plantea Nassif, Sabor decide desarrollar el que se refiere a los planes y programas de enseñanza, en el entendido de que es necesario clarificar los límites actuales y futuros de la disciplina para luego discutir cómo enseñarla. En este sentido, Sabor señala:

la aparición y el desarrollo de la documentación es lo que crea las mayores dudas, al colocar en situación de crisis el criterio tradicional que fija a la bibliotecología límites que ahora resultan estrechos y que ponen de manifiesto lo limitado de los medios y formas tradicionales de trabajo de las bibliotecas frente a la complejidad de las nuevas situaciones (Sabor, 1968, p.69).

Cita los aportes de diversos teóricos (Rees, Saracevic, Shera, Shaw) para preguntarse si la documentación es una nueva disciplina o si solo viene a enriquecer la bibliotecología tradicional. A pesar de que la autora considera que sería útil que las escuelas crecieran en interdisciplinariedad y formaran documentalistas, ve como inquietante la implementación de nuevas estructuras en países que aún no cuentan con las condiciones necesarias. Al considerar que los factores económicos y culturales del medio tienen gran influencia en la formación universitaria, Sabor decide que sus propuestas estén dirigidas únicamente a escuelas de bibliotecarios en países en vías de desarrollo. A pesar de ello, acuerda con Morton (1961) cuando este afirma:

Debemos formar bibliotecarios para el cambio. Las bibliotecas de hoy son muy distintas de las que había cuando muchos de ustedes y yo misma ingresamos en la profesión. Mañana no serán lo que son hoy. Los hechos, las prácticas que enseñamos hoy pueden quedar anticuados antes de que termine sus estudios la promoción de este año. Debemos enseñar principios generales, desarrollar en los estudiantes la capacidad de hacer por sí mismos adaptaciones y aplicaciones, con la visión y la imaginación necesarias para hacer frente a los cambios previsibles y que encontrarán sin duda durante su carrera profesional" (Morton 1961, citado por Sabor, 1968, p.73).

Ríos (2008,2015) coincide con Emilio Setién Quesada quien en el III Encuentro EDIBCIC (1996) recopiló información de los planes de estudio de las escuelas latinoamericanas de Bibliotecología. En ese momento, el investigador cubano señalaba la necesidad de identificar qué concepciones subyacen a la elaboración de esas currículas identificando un conflicto entre una formación generalista y una que pretendía adecuarse continuamente a las exigencias del mercado laboral. En ese sentido, Ríos apunta que las transformaciones de la disciplina bibliotecológica a finales del siglo $\mathrm{XX}$, se reflejaron, en la mayoría de las escuelas de Latinoamérica, en actualizaciones parciales de planes y programas que no exigieron grandes modificaciones estructurales y de contenido. La presencia en aumento de aspectos teóricos en programas que estaban inclinados en el pasado a la enseñanza predominante de técnicas y procedimientos, podría leerse como 
positivo. Sin embargo, Ríos advierte:

Sin embargo, enfatizar las teorías bibliotecológicas a través de asignaturas introductorias no modifica en lo sustancial la organización orientada a las áreas profesionales de la disciplina que se da en las escuelas. Lo cual ha implicado que la pretensión de enseñar más teoría bibliotecológica de carácter explicativa no se cumpla porque las asignaturas destinadas a ello están subordinadas al peso académico del área profesional (Ríos, 2008, p.29).

\section{Conclusiones}

Este trabajo optó por analizar los aportes de algunas de las voces más importantes en el ámbito de la Bibliotecología y la Ciencia de la Información de Latinoamérica. Sabor y Nassif hicieron sus planteos más destacables en 1968, siendo impulsados por la edición que realizó Unesco de su manual. Mientras tanto, el mexicano Jaime Ríos, comenzó cuarenta años después una prolífica teorización en pos de generar una didáctica específica de nuestra disciplina. A pesar de que nos separan cincuenta años de la publicación de Sabor y Nassif, podemos comprobar que muchas de sus inquietudes siguen vigentes en los textos de Ríos. Es de destacar que varios de los aspectos que ellos desarrollaron siguen siendo objeto de estudio de docentes e investigadores de nuestro continente y eso se refleja tanto en los regulares encuentros de EDICIC como en la realidad cotidiana de nuestras aulas.

Las tres tensiones que atraviesan a la enseñanza universitaria de la Bibliotecología encuentran en estos docentes e investigadores distintas respuestas. Se considera útil señalar las coincidencias y disidencias identificadas en el análisis para arribar a una conclusión final.

La primera tensión se centraba en distinguir si la Universidad debe hacer mayor énfasis en la formación profesional o priorizar su rol como centro de producción de conocimiento. Tanto Sabor como Nassif consideran de fundamental importancia la formación para el futuro desempeño profesional. Sin embargo, consideran que los estudiantes deben desarrollar una actitud crítica y creativa y recibir una fuerte base humanista, para no terminar siendo simples operarios sin capacidades resolutivas ni generadoras de nuevas realidades. Para ello, Nassif propone que la universidad problematice sus contenidos, abandonando la visión clásica de la enseñanza como un "cuerpo rígido de métodos" que impone al alumno "un saber elaborado de antemano" en cuya elaboración ni docente ni estudiante ha participado (Nassif, 1968:37). Las nuevas perspectivas que sobre el aprendizaje se estaban desarrollando en esos años, permitían hacer foco en el proceso que el estudiante vivía en el aula cuando en ella se permitía la construcción, la discusión y la ejecución de experiencias. Nassif identifica una energía creadora en los docentes y estudiantes que debe alimentarse con la existencia de espacios de formación que se nutren del encuentro del científico que comparte su práctica investigativa con un estudiante que se asocia. 
Los aportes de Ríos van en el mismo sentido. A pesar de que este investigador hace mayor hincapié en la formación teórica de los estudiantes, no desconoce que ellos deben salir preparados para el mundo laboral, pues no se busca crear únicamente investigadores, sino profesionales capaces de analizar y solucionar los problemas sociales de la realidad fuera de las aulas. Para ello se permite distinguir dos niveles de teorización de la Bibliotecología como disciplina científica. Así, la teoría prescriptiva puede relacionarse, en su nivel básico, con las facetas más pragmáticas del ejercicio profesional; y en su nivel más complejo, con prescripciones comprensivas, que apuntan a su utilidad social. Por otro lado, la teoría explicativa se enfoca en la construcción de un corpus conceptual que permita explicar los fenómenos que son su objeto de estudio. Ambas teorías no pueden hacerse carne en una práctica desprovista de racionalidad y espíritu crítico. Esta construcción disciplinar que reconoce la naturaleza teórica de la Bibliotecología, permitiría abandonar la mirada que piensa en ella como centrada en el "oficio"; y por lo tanto, abre el camino para que las escuelas donde se enseña promuevan la creación de nuevos conocimientos. Igualmente, vale señalar que los esfuerzos de Ríos por instalar una didáctica específica de la disciplina se alimentan de décadas de discusión y crecimiento que han allanado el camino hacia una episteme propia. Por su parte, en el momento histórico que teoriza Sabor, está asomando el concepto de documentación acompañado de la posibilidad de implementación de nuevas estructuras en los centros de estudio y en los lugares de trabajo. La docente prioriza, entonces, la realidad de su país y considera que la formación, en ese momento, debe centrarse en formar bibliotecarios para trabajar en bibliotecas. Por su parte, , Nassif augura que la enseñanza universitaria debe preparar al sujeto para que adquiera autónomamente los nuevos conocimientos que ya en esos años, crecían de manera inusitada y diversificada.

La segunda tensión se centraba en la discusión sobre si es necesaria una formación en aspectos didácticos y pedagógicos de los profesores universitarios. En este aspecto, tanto Sabor, como Nassif y Ríos coinciden en afirmar que sí lo es. Sabor y Nassif señalan la escasa preparación que tienen los docentes en aspectos didáctico y su libro, en definitiva, fue un intento de responder a las necesidades planteadas desde las aulas. Nassif plantea que la pedagogía universitaria debe dejar su carácter empírico e intuitivo para pasar a ser científica y sistemática. Ríos también muestra una fuerte preocupación sobre la falta de formación de los docentes, tanto en los aspectos disciplinares como en los pedagógicos.

Sobre los procedimientos de enseñanza, las miradas de Sabor y Nassif comparten un enfoque fuertemente instrumental, pero esto también responde al tipo de publicación que estamos analizando que se configura como un manual. Mientras tanto, Ríos hace énfasis en que el desarrollo de los fundamentos de una didáctica específica depende de las categorías de racionalidad de la ciencia bibliotecológica, por lo que sus propuestas son quizás más abstractas. El método de enseñanza que acompañaría esta idea de Ríos es la que propone Nassif como forma más elevada. En el seminario 
es donde el aprendizaje de la disciplina se logra mediante la aplicación del método específico que ella posee. Estos espacios de aprendizaje promueven la capacidad de análisis, evaluación y contrastación de argumentos y pueden equipararse a los nichos cognitivos que plantea Ríos en 2015. Para el investigador mexicano, el desarrollo de estas competencias en el estudiante y futuro profesional alimentará la creación de nuevas teorías disciplinares. Por otro lado, Nassif y Ríos coinciden en su preocupación sobre los procesos de aprendizajes, y promueven las iniciativas donde el centro se corre del maestro hacia el alumno. La aplicación de ese método no se visualiza como una habilidad únicamente procedimental, sino como manera de pensar el mundo y de construir explicaciones. Otra preocupación que comparten Nassif y Ríos es la necesaria revisión de las prácticas de enseñanza que los docentes llevan adelante en las aulas, tanto sea para rescatar aquellas experiencias exitosas como para poner en cuestión aquello que se aprendió y resultó en prácticas arraigadas que no admiten cuestionamientos. Un factor que los tres autores identifican como problemático es que, en muchas escuelas, el estudiante que se gradúa pasa rápidamente al ejercicio docente, sin contar con formación en pedagogía y didáctica.

La tercera tensión se centraba en la presencia de los paradigmas disciplinares al momento de diseñar planes y programas. En este aspecto, los tres autores coinciden en la necesidad de clarificar los límites actuales y futuros de la disciplina para luego discutir cómo enseñarla. A pesar de ello, Sabor y Nassif hacen mayor énfasis en la relación que la disciplina tiene con la realidad, mientras que Ríos plantea la urgencia de construir una episteme fuerte, que no responda sistemáticamente a los devenires de la realidad. Es necesario "organizar los currículos a partir de una concepción integral de la disciplina y sus fenómenos" (Ríos, 2008, p.27-28), más que por las transformaciones de la profesión. En el diseño del currículo, esto no significa necesariamente el aumento de asignaturas teóricas si estas siguen supeditadas a la formación de corte pragmático.

En definitiva, Sabor, Nassif y Ríos consideran necesario instalar una didáctica específica para la enseñanza de la Bibliotecología. Si bien los primeros responden a la realidad de su tiempo y hacen mayor énfasis en los procedimientos didácticos (cómo llevar adelante una clase), coinciden con Ríos en que la problematización de los contenidos en el aula garantiza una comunidad de trabajo que favorezca la generación de nuevos conocimientos.

Coinciden también en la necesaria discusión que debe darse sobre el peso de los conocimientos teóricos y prácticos al momento de generar nuevos currículums y sobre la relación ulterior que ellos tienen en el desempeño profesional. Nuevamente, el contexto histórico influye en que Sabor decida hacer énfasis en una formación que apunta a que sus futuros egresados trabajen en bibliotecas en países en vías de desarrollo. La mirada más contemporánea de Ríos, ve que la realización profesional requiere que los estudiantes se formen sólidamente en aspectos teóricos, ya que eso será lo que les permitan enfrentar los problemas prácticos que se presentan en el 
campo profesional de forma más crítica y creativa, sea cual fuera su lugar de desempeño. Sin embargo, podemos afirmar que ambos coinciden en la necesidad de una formación integral de base humanista, pues esta alimenta una actitud flexible y ética en los futuros profesionales.

Por último, los tres autores ven en la escasa formación pedagógica de los docentes universitarios en general y de Bibliotecología en particular, un importante obstáculo para mejorar tanto la enseñanza como el aprendizaje de esta disciplina.

\section{Referencias bibliográficas}

BASABE, L. (2016). Acerca de los usos de la teoría didáctica. En

A.Camilloni (comp), El saber didáctico, pp. 201-231. Buenos Aires: Paidós.

BEHARES, L. (2011). Enseñanza y producción de conocimiento: la noción de la enseñanza en las políticas universitarias uruguayas. Montevideo:

Universidad de la República.

CAMILlONI, A. (2007). Los profesores y el saber didáctico. En A. Camilloni (comp.), El saber didáctico, pp. 41-60. Buenos Aires: Paidós.

DAVINI, M.C. (1998). La formación docente en cuestión: política y pedagogía. Buenos Aires: Paidós.

DÍAZ BARRIGA, A. (1997). Didáctica y currículum: convergencias en los programas de estudio. Buenos Aires: Paidós.

FERREIRA, M.M, BOTTENTUIT, A.M. (2017). Didática na Biblioteconomia: reflexões sobre a prática de professores na disciplina Fundamentos de Biblioteconomia da UFMA. REBECIN [en línea], 4 (n. esp.), pp. 19-36. [Consultado: 14 febrero 2018]. Disponible en: http://abecin.org.br/portalerevistas/index.php/rebecin

GARCÍA-VALCÁRCEL, A. (2001). Didáctica Universitaria. Madrid: La Muralla, 2001.

LITWIN, E. (1998a). El campo de la didáctica: la búsqueda de una nueva agenda. En A. Camilloni, et. al. Corrientes didácticas contemporáneas, pp. 91-115. Buenos Aires: Paidós.

LITWIN, E. (1998b). La didáctica: una construcción desde la perspectiva de la investigación en el aula. Educación [en línea], VII (13), pp. 41-59. [Consultado: 14 febrero 2018]. Disponible en: https://dialnet.unirioja.es/descarga/articulo/5056779.pdf

MENESES TELLO, F. (2010). La educación bibliotecológica: tema de estudio en el pensamiento de Judith Licea. Información, cultura y sociedad [en línea], (23), pp. 9- 30. [Consultado: 14 febrero 2018]. Disponible en: http://www.scielo.org.ar/scielo.php? script=sci_arttext\&pid=S1851$17402010000200002 \& \operatorname{lng}=\mathrm{es} \& \operatorname{tlng}=\mathrm{es}$ 
NASSIF, R. (1968). Fundamentos de pedagogía y metodología: Estudio preliminar para una pedagogía bibliotecológica. En Josefa Sabor, Métodos de enseñanza de la bibliotecología, pp.15-62. París: UNESCO, 1968.

NÚÑEZ ANSUAS, J. (2018). Prácticas de enseñanza en Ciencia de la Información : aportes de los Encuentros de la Asociación de Educación e Investigación en Ciencia de la Información de Iberoamérica y el Caribe (EDICIC) desde sus orígenes hasta el presente (Trabajo final de grado). Facultad de Información y Comunicación, Montevideo, Uruguay.

RÍOS, J. (2008). Didáctica de la Bibliotecología: Teoría y principios desde la enseñanza de la ciencia. México : UNAM, Centro Universitario de Investigaciones Bibliotecológicas.

RÍOS, J., PULIDO, N. (coord). (2015). Didáctica de la bibliotecología: aproximaciones e instituciones. México: UNAM, Instituto de Investigaciones Bibliotecológicas y de la Información.

RÍOS, J. (2018). Vindicación de la didáctica de la bibliotecología: construir la racionalidad y el sentido en una disciplina compleja [aún no publicada]. En: EDIBCIC XI (2018). XI Encuentro de Educadores e Investigadores de Bibliotecología, Archivología y Ciencia de la Información en Iberoamérica y el Caribe: Tendencias en Ciencias de la Información. Medellín: Universidad de Antioquia. 16-19 octubre 2018.

ROSALES LÓPEZ, C. (2001). Comunicación didáctica en la universidad. En: A. García Valcárcel. Didáctica Universitaria, pp.113-151. Madrid: La Muralla.

SABOR, J. (1968). Métodos de enseñanza de la bibliotecología. París: UNESCO.

SANTOS, B. S. (2010). Descolonizar el saber, reinventar el poder. Montevideo: Trilce.

SETIÉN QUESADA, E. (1996). Documento de Trabajo B, 4a, “Tendencias de la formación bibliotecológica en la América Latina”. En: EDIBCIC III (1996). III Encuentro de Educadores e Investigadores de Bibliotecología, Archivología y Ciencia de la Información en Iberoamérica y el Caribe [actas]. San Juan : Universidad de Puerto Rico. 11-17 agosto 1996.

STOKER, D. (2000). Persistence and change:Issues for LIS educators in the first decade of the twenty first century. Education for information, 18(2/3), pp. $115-122$.

ZABALZA, M. Á. (2002). La enseñanza universitaria:el escenario y sus protagonistas. Madrid: Narcea Ediciones.

ZABALZA, M. Á. (2007). La Didáctica Universitaria. Bordón. Revista de pedagogía, 59, (2-3), p. 489-510. 